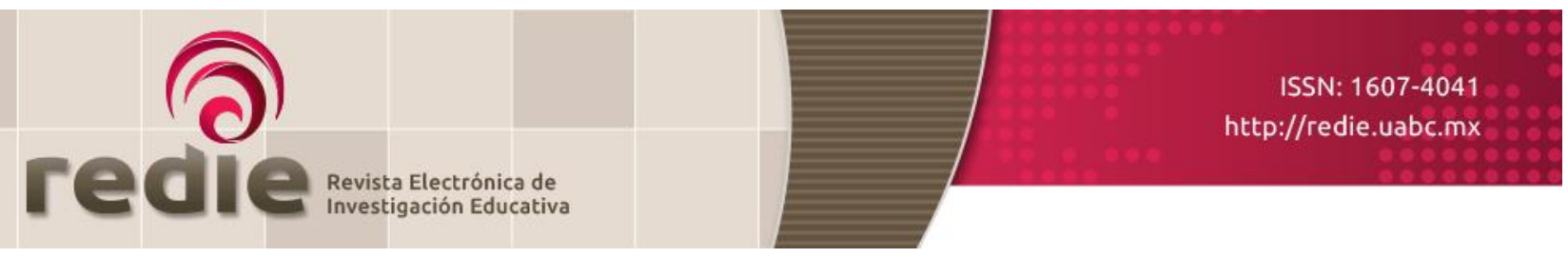

Vol. 19, Núm. 1, 2017

\title{
Estudio de la ansiedad matemática en la educación media costarricense
}

\section{Mathematical Anxiety in Secondary Education in Costa Rica}

\author{
Evelyn Agüero Calvo (*) evaguero@itcr.ac.cr \\ Luis Gerardo Meza Cascante $\left(^{*}\right)$ gemeza@itcr.ac.cr \\ Zuleyka Suárez Valdés-Ayala $\left.*^{*}\right)$ zsuarez@itcr.ac.cr \\ Sandra Schmidt Quesada $\left(^{*}\right)$ sschmidt@itcr.ac.cr \\ (*) Instituto Tecnológico de Costa Rica \\ (Recibido: 6 de agosto de 2014; Aceptado para su publicación: 9 de octubre de 2015)
}

Cómo citar: Agüero, E., Meza, L. G., Suárez, Z. y Schmidt, S. (2017). Estudio de la ansiedad matemática en la educación media costarricense. Revista Electrónica de Investigación Educativa, 19(1), 35-45. Recuperado de https://doi.org/10.24320/redie.2017.19.1.849

\section{Resumen}

En este artículo se presentan los resultados de un análisis del nivel de "ansiedad matemática", entendida como la ausencia de comodidad que alguien podría experimentar cuando se le exige tomar clases de matemática, tal como lo muestran los estudiantes que cursan educación secundaria en Costa Rica. La investigación se realizó con una muestra de 3,725 estudiantes de educación secundaria pública en Costa Rica, en el año 2013. Los resultados revelan diferencias estadísticamente significativas en el nivel de ansiedad matemática por género, teniendo las mujeres niveles moderadamente más altos de ansiedad. Las diferencias en los niveles de ansiedad también están determinadas por el nivel educativo que cursa el estudiante, se encontraron diferencias entre los estudiantes del tercer ciclo y los de educación diversificada, estos últimos mostraron niveles de ansiedad moderadamente más altos. Sin embargo, el estudio concluye que a pesar de las diferencias observadas, sólo un $22.4 \%$ de los estudiantes muestra niveles de ansiedad matemática altos o muy altos.

Palabras clave: Enseñanza secundaria, matemáticas, afectividad, ansiedad.

\section{Abstract}

This paper offers the results of an analysis of the level of mathematical anxiety, understood to mean a feeling of discomfort experienced when someone is required to take mathematics classes, as displayed in students of secondary education in Costa Rica. The study was performed with a sample of 3,725 students in public secondary education in Costa Rica in 2013. The results show statistically significant differences between the sexes in the level of mathematical anxiety, with female students experiencing moderately higher anxiety levels. Differences in anxiety levels are also determined by the student's educational level, with significant differences found between third-cycle students $\left(7^{\text {th }}, 8^{\text {th }}\right.$ and $9^{\text {th }}$ grades) and diversified education students $\left(10^{\text {th }}\right.$ and $11^{\text {th }}$ grades); the latter displayed moderately higher anxiety levels. Nonetheless, the study concludes that despite the differences observed, only $22.4 \%$ of students show 
high or very high levels of mathematical anxiety.

Keywords: Secondary education, mathematics, affectivity, anxiety.

\section{Introducción}

En los últimos tiempos hemos pasado de una sociedad que empleaba los símbolos gráficos para leer y escribir, a un nuevo modelo cultural que renueva el concepto de alfabetización, llevándolo más allá de la lectura y escritura textual, y que exige el dominio del lenguaje audiovisual y multimedia. La Universidad, en este sentido, se ve obligada a asumir una tarea alfabetizadora que haga posible el acceso igualitario a la tecnología y el desarrollo de una ciudadanía responsable y crítica (Área, Gutiérrez y Vidal, 2012). En la era de la información y el conocimiento, la alfabetización digital es un derecho del individuo.

La comunidad investigadora es consciente de la influencia de los factores afectivos en el aprendizaje de la matemática y, por este motivo, en los últimos años se ha incrementado el número de trabajos que profundizan en este aspecto (Gómez-Chacón, 2010).

McLeod (1989), citado por Gómez-Chacón (2000, p. 186), considera que el dominio afectivo es "un extenso rango de estados de ánimo que son generalmente considerados como algo diferente de la pura cognición" e incluye, como componentes específicos, creencias, actitudes y emociones. En la literatura especializada se suele denominar a estos tres componentes específicos como descriptores básicos del componente afectivo del aprendizaje de la matemática (aunque algunos autores añaden los valores como cuarto descriptor básico). La ansiedad matemática, tal como señalan Pérez-Tyteca y Castro (2011), con base en los estudios de Hart (1989) y Evans (2000), es considerada por diversos autores como una actitud.

No existe unicidad en cuanto a la definición de ansiedad matemática. Pérez-Tyteca y Castro (2011) resaltan que las definiciones de ansiedad matemática suelen asumir implícitamente dos hechos. Por una parte, que la ansiedad matemática está relacionada con la ansiedad general (Hendel, 1980), con la ansiedad hacia los exámenes y con la producida por otras materias académicas; sin embargo, por otra parte, también es específica (Hembree, 1990), es decir, la ansiedad matemática existe en personas que no tienen otros tipos de ansiedad (Morris,1981).

En la investigación se asumió el constructo ansiedad matemática como aquel estado afectivo que se caracteriza por la ausencia de confort que puede experimentar una persona en situaciones relacionadas con las matemáticas, tanto de su vida cotidiana como académica, y que se manifiesta mediante un sistema de respuestas que engloban una serie de síntomas, como son: tensión, nervios, preocupación, inquietud, irritabilidad, impaciencia, confusión, miedo y bloqueo mental (Pérez-Tyteca, Monje y Castro, 2013).

Aunque el estudio de la ansiedad matemática se inició hace más de 40 años, sigue siendo un tema de actualidad (Pérez-Tyteca, Monje y Castro, 2013). Prueba de ello es la inclusión de esta variable por parte de la Organización para la Cooperación y el Desarrollo Económicos (OCDE) en el estudio PISA 2003 implementado en 40 países, en el que se ha comprobado que una gran proporción de alumnos de 15 años manifiesta sentimientos de inseguridad y estrés emocional cuando se enfrenta a la matemática (PérezTyteca, Monje y Castro, 2013).

Según el estudio de la OCDE (Pérez-Tyteca et al. 2013), los estudiantes que sienten ansiedad cuando estudian matemática tienden a no interesarse en el estudio de la disciplina y no disfrutar de ella, encontrándose una relación consistente en todos los países estudiados, lo que constituye un patrón de relaciones universal (OCDE, 2004).

Se ha encontrado relación entre el nivel de ansiedad matemática y la autoconfianza: los alumnos con más ansiedad presentan menor confianza en sus habilidades matemáticas, dándose una relación negativa, como han mostrado Bursal y Paznokas (2006) e Isiksal, Curran, Koc y Askun (2009), como se cita en PérezTyteca y Castro (2011). 
Investigaciones realizadas por Pérez-Tyteca et al. (2013) han mostrado que existe correlación negativa entre el nivel de ansiedad matemática y la autoconfianza en los estudiantes que realizan el paso de la educación secundaria a la universitaria, y se producen en ambos constructos diferencias significativas de género a favor de los hombres, lo que tiene efecto sobre la elección de la carrera.

También se ha estudiado la relación entre la ansiedad matemática y el género, temática en la que los resultados no son concluyentes. En efecto, en algunos estudios se ha comprobado la existencia de una tendencia que lleva a las mujeres a reportar mayor ansiedad matemática que los hombres, tales como los realizados por Valero (1999) y Gil, Blanco y Guerrero (2006), entre otros. Resultados similares son reportados por Fennema y Sherman (1976), Lafferty (1994), Vest (1998), Wigfield y Meece (1988) y Worley (1997), citados por Pérez-Tyteca y Monje (2013). Pero también hay estudios que no han encontrado diferencias significativas en el nivel de ansiedad según el género.

Tal como indican Tejedor, Santos, García-Orza, Carratalá y Navas (2009), una de las consecuencias más importantes de la ansiedad matemática es que las personas con mayores niveles de ansiedad matemática muestran tendencia a evitar los programas formativos en los que se imparte la materia. Según estos autores, con fundamento en Kirschner, DiRita y Flynn (2005), las personas con niveles altos de ansiedad matemática suelen mostrar actitudes negativas hacia las tareas de tipo aritmético y tienen un autoconcepto negativo en cuanto a su ejecución en las mismas.

Aunque la búsqueda de las causas que provocan la ansiedad hacia las matemáticas es a menudo infructuosa, tal como indican Muñoz y Mato (2007), el interés de la investigación se justifica porque dentro de las consecuencias de sentir ansiedad matemática están: la disminución del éxito en esta materia, la evasión de matricularse en cursos que requieran de esa asignatura, la limitación a la hora de escoger una carrera universitaria, y el surgimiento de sentimientos negativos de culpa y vergüenza.

Con base en resultados como los obtenidos por Ashcraft y Kirk (2001), como se cita en Tejedor et al. (2009), se puede decir que las personas con ansiedad alta hacia la matemática no son intelectualmente menos capaces que los demás, pero ante tareas de tipo aritmético se consideran peores y evitan afrontarlas.

El objetivo general de la investigación fue estudiar el nivel de ansiedad matemática de los estudiantes de la educación media costarricense; con los propósitos específicos de determinar el nivel de ansiedad matemática de los estudiantes de la educación media, establecer si existen diferencias significativas entre hombres y mujeres respecto a la ansiedad matemática que manifiestan los estudiantes de la educación media e identificar los niveles educativos que presentan un mayor nivel de ansiedad matemática entre sus estudiantes.

\section{Metodología}

La investigación es de enfoque cuantitativo, clasificable como descriptiva, cuya población de interés estuvo compuesta por estudiantes de educación media costarricense matriculados en colegios académicos oficiales diurnos. La selección de los colegios participantes se realizó a partir de un listado de colegios oficiales, tomado de la página del Ministerio de Educación Pública. De ese marco muestral se seleccionaron aleatoriamente cinco centros educativos de cada provincia y dentro de cada uno de ellos un grupo de cada nivel. La muestra estuvo integrada por 3,725 estudiantes (48.1\% mujeres y $51.9 \%$ hombres).

En la investigación se consideraron tres variables: sexo, nivel educativo y ansiedad matemática, cuya definición conceptual y operativa se muestra en la tabla I. 
Tabla I. Definición conceptual y operativa de las variables

\begin{tabular}{lll}
\hline Variable & Definición conceptual & Definición operativa \\
\hline Sexo & $\begin{array}{l}\text { Condición de masculino o } \\
\text { femenino. }\end{array}$ & $\begin{array}{l}\text { Según el estudiante indique } \\
\text { en el formulario aplicado. }\end{array}$ \\
\hline Nivel educativo & $\begin{array}{l}\text { Nivel de educación media } \\
\text { que cursa el o la estudiante, } \\
\text { a saber: séptimo, octavo, } \\
\text { noveno, décimo o undécimo. }\end{array}$ & $\begin{array}{l}\text { Según el estudiante indique } \\
\text { en el formulario aplicado. }\end{array}$ \\
\hline Ansiedad matemática & $\begin{array}{l}\text { Ausencia de confort que } \\
\text { alguien podría experimentar } \\
\text { cuando se le exige rendir en } \\
\text { matemáticas. }\end{array}$ & $\begin{array}{l}\text { Puntaje obtenido por el } \\
\text { estudiante mediante la } \\
\text { aplicación de la Escala de } \\
\end{array}$ \\
& $\begin{array}{l}\text { Ansiedad Matemática de } \\
\text { Fennema y Sherman (1976). }\end{array}$ \\
\hline
\end{tabular}

Fuente: Elaboración propia.

Como instrumento de medición se utilizó la "Escala de Ansiedad Matemática" de Fennema y Sherman (1976), por ajustarse al concepto de ansiedad matemática asumido en esta investigación y haber sido validada a lo largo de 30 años de aplicación. Este instrumento consiste en un cuestionario tipo Likert integrado por 12 ítems, en los cuales existen cinco posibilidades de respuesta que van desde "Totalmente de acuerdo" a "Totalmente en desacuerdo", con la opción central o neutra "Indeciso".

En cada grupo se aplicó el instrumento de medida al total de estudiantes presentes el día de la aplicación del cuestionario. Para evidenciar la validez del instrumento de medición se realizó un estudio del índice de discriminación de cada ítem que integra la escala, mediante el cálculo de la correlación entre la puntuación obtenida en el ítem y la obtenida en el instrumento (excluyendo la puntuación correspondiente al ítem, para no incrementar de manera artificial el valor de la correlación entre ambas puntuaciones) (Lozano y De la Fuente, 2009).

La interpretación del valor de los índices de discriminación se hizo con base en los intervalos de baremación propuestos en Lozano y De la Fuente (2009, p. 12) que se muestran en la tabla II.

Tabla II. Rangos de valoración de los índices de discriminación

\begin{tabular}{l|l}
\hline Valores & Interpretación \\
\hline Igual o mayor que 0.40 & El ítem discrimina muy bien \\
Entre 0.30 y 0.39 & El ítem discrimina bien \\
Entre 0.20 y 0.29 & El ítem discrimina poco \\
Entre 0.10 y 0.19 & Ítem límite. Se debe mejorar \\
Menor de 0.1 & El ítem carece de utilidad para discriminar \\
\hline
\end{tabular}

Fuente: Lozano y De la Fuente (2009, p.12).

También se procedió evidenciar la unidimensionalidad, es decir, a mostrar que el instrumento mide sólo un rasgo o constructo que, para efectos de la investigación, corresponde al constructo de ansiedad matemática.

La unidimensionalidad del instrumento se estudió mediante el análisis factorial exploratorio por ser la técnica más utilizada para estos propósitos (Jiménez y Montero, 2013), constatando el cumplimiento de al menos uno de los siguientes criterios:

La estructura de un factor dominante tras el análisis de los autovalores (Arias, 1996, como se cita en Rivas, Fierro, Jiménez y Berrocal, 1998):

$$
\frac{\lambda_{1}}{\lambda_{2}}>5 \quad \circ \quad \frac{\lambda_{1}-\lambda_{2}}{\lambda_{2}-\lambda_{3}}>5
$$


El primer factor explica el 40\% de la varianza (Carmines y Zeller, 1979, como se cita en Burga, 2006).

Para verificar la razonabilidad de aplicar el análisis factorial se efectuó la Prueba de esfericidad de Bartlett y se calculó el Índice de adecuación muestral de Kaiser-Meyer y Olkin (KMO).

Para estudiar la confiabilidad del instrumento (la fiabilidad de la escala) se utilizó el alfa de Cronbach, siguiendo la recomendación de Cea (1999), de que un valor mínimo de 0.8 es adecuado.

El análisis estadístico descriptivo de los datos incluyó la baremación de los puntajes de la escala con el propósito de establecer una distribución de la muestra en cuatro categorías siguiendo la interpretación de los puntajes de acuerdo con lo planteado por Pérez-Tyteca (2012): identificar valores cercanos a 1 con un nivel muy bajo de ansiedad matemática, valores en torno a 2 con un nivel bajo, valores que rondan el 3 como un nivel medio, los próximos a 4 con un nivel alto y los valores situados alrededor de 5 con un nivel muy alto de ansiedad matemática.

Posteriormente, se procedió al contraste de las hipótesis. Para el caso de la hipótesis relacionada con las diferencias por género (Hipótesis 1) se utilizó la prueba paramétrica $t$ de Student, asumiendo la normalidad de la distribución de los datos y la prueba no paramétrica de U de Mann-Whitney. Para la hipótesis relacionada con el nivel educativo (Hipótesis 2) se utilizó la prueba ANOVA complementada con la prueba a posteriori de Bonferroni.

Por último, se calculó el tamaño del efecto para los casos en que se detectó la existencia de diferencias estadísticamente significativas utilizando la $d$ de Cohen (Ripoll, 2011), que representa el número de desviaciones típicas que separan a dos grupos.

Para la interpretación de la magnitud de los tamaños del efecto medidos por la $d$ de Cohen se suelen utilizar las referencias dadas por Cohen (Morales, 2008 y Ripoll, 2011): en torno a 0.20 (diferencia pequeña), en torno a 0.50 (diferencia moderada) y 0.80 o más (diferencia grande).

\section{Resultados}

\subsection{Estadísticos descriptivos}

En la investigación participaron estudiantes de la educación media costarricense matriculados durante el año 2013 en colegios públicos. La muestra estuvo integrada por 3,725 estudiantes, según la distribución por provincia, sexo y nivel educativo que se muestra en las tablas III, IV y V.

Tabla III. Distribución de la muestra por provincia

\begin{tabular}{lcc}
\hline Provincia & Frecuencia & Porcentaje \\
\hline Alajuela & 533 & 14.3 \\
Cartago & 607 & 16.3 \\
Guanacaste & 406 & 10.9 \\
Heredia & 425 & 11.4 \\
Limón & 480 & 12.9 \\
Puntarenas & 553 & 14.8 \\
San José & 721 & 19.4 \\
\hline Total & 3725 & 100 \\
\hline
\end{tabular}

Fuente: Elaboración propia. 
Tabla IV. Distribución de la muestra por sexo

\begin{tabular}{l|c|c}
\hline Sexo & Frecuencia & Porcentaje \\
\hline Mujeres & 1791 & 48.1 \\
Hombres & 1934 & 51.9 \\
\hline Total & 3725 & 100 \\
\hline
\end{tabular}

Fuente: Elaboración propia.

Tabla V. Distribución de la muestra por nivel educativo

\begin{tabular}{lcc}
\hline Nivel & Frecuencia & Porcentaje \\
\hline Séptimo & 745 & 20.0 \\
Octavo & 742 & 19.9 \\
Noveno & 773 & 20.8 \\
Décimo & 744 & 20.0 \\
Undécimo & 721 & 19.4 \\
\hline Total & 3725 & 100 \\
\hline
\end{tabular}

Fuente: Elaboración propia.

\subsection{Resultados según la clasificación del nivel de ansiedad}

El nivel medio de ansiedad matemática ( $M=33.22$, $D T=9.76)$, fue significativamente menor que el promedio de la escala $(T(3725)=188.88, \mathrm{p}<0.05)$.

De esta manera, globalmente los estudiantes de la muestra presentan un nivel de ansiedad matemática ligeramente inferior al nivel medio, lo que indica que no se sienten excesivamente ansiosos.

Con la baremación de los resultados del instrumento en las cuatro categorías indicadas, se realizó una clasificación de la muestra como se observa en la tabla VI.

Tabla VI. Clasificación del nivel de ansiedad

\begin{tabular}{l|c|c|c}
\hline Categoría & Frecuencia & Porcentaje & $\begin{array}{c}\text { Porcentaje } \\
\text { acumulado }\end{array}$ \\
\hline Muy bajo & 272 & 7.3 & 7.3 \\
Bajo & 1092 & 29.3 & 36.6 \\
Medio & 1528 & 41.0 & 77.6 \\
Alto & 759 & 20.4 & 98.0 \\
Muy alto & 74 & 2.0 & 100 \\
\hline Total & 3725 & 100 & \\
\hline
\end{tabular}

Fuente: Elaboración propia.

Estos resultados indican que aproximadamente el $37 \%$ de los estudiantes presenta niveles de ansiedad matemática bajo o muy bajo y cerca de un $78 \%$ presenta niveles medios de ansiedad matemática o rangos menores.

\subsection{Resultados asociados al instrumento de medición}

Índice de discriminación de los ítems. Siguiendo la clasificación de Lozano y De la Fuente (2009, p. 12), todos los ítems discriminan bien, pues su índice de discriminación supera el valor de 0.3, con excepción del número 2. Por lo que, eliminando el ítem 2 y volviendo a calcular el índice de discriminación para los 11 ítems restantes, se obtienen los resultados de la tabla VII. 
Tabla VII. Índice de discriminación de los ítems

\begin{tabular}{c|c}
\hline Ítems & $\begin{array}{c}\text { Índice de } \\
\text { discriminación }\end{array}$ \\
\hline 1 & 0.486 \\
3 & 0.353 \\
4 & 0.488 \\
5 & 0.641 \\
6 & 0.550 \\
7 & 0.679 \\
8 & 0.649 \\
9 & 0.513 \\
10 & 0.633 \\
11 & 0.693 \\
12 & 0.746 \\
\hline \multicolumn{2}{|c}{ Fuente: Elaboración propia. }
\end{tabular}

Dado que todos los valores superan 0.3 puede considerarse que los ítems muestran un adecuado nivel de discriminación. Consecuentemente, los análisis restantes se realizaron a partir de los valores arrojados por los 11 ítems con adecuado índice de discriminación.

Confiabilidad del instrumento. El valor del alfa de Cronbach para el instrumento utilizado fue de 0.876 , razón por la cual, siguiendo el criterio de Cea (1999), se tiene que el instrumento mostró una adecuada confiabilidad.

Unidimensionalidad del instrumento. Para evidenciar la razonabilidad de aplicar el análisis factorial, se calcularon el índice Kaiser-Meyer-Olkin (KMO) y la prueba de esfericidad de Bartlett, con los resultados mostrados en la tabla VIII.

Tabla VIII. KMO y prueba de esfericidad de Bartlett

\begin{tabular}{l|l|l}
\hline \multicolumn{2}{l|}{ Medida de adecuación muestral de KMo } & 0.922 \\
\cline { 2 - 3 } $\begin{array}{l}\text { Prueba } \\
\text { de esfericidad } \\
\text { de Bartlett }\end{array}$ & Chi-cuadrado aproximado & 16126.773 \\
Fuente: Elaboración propia. & Sig. & 55 \\
\hline
\end{tabular}

El valor de 0.922 para el índice KMo indica una excelente adecuación muestral. Por otra parte, el valor $p<0.001$ en el índice de Bartlett, también indica que es adecuado desarrollar un análisis factorial con estos datos. 
Al realizarse el análisis factorial se obtuvieron los datos que se muestran en la tabla IX.

Tabla IX. Resultados del análisis factorial

\begin{tabular}{c|c|c|c}
\hline \multirow{2}{*}{ Componente } & \multicolumn{3}{|c}{ Autovalores iniciales } \\
\cline { 2 - 4 } & Total & $\begin{array}{c}\text { \% de la } \\
\text { varianza }\end{array}$ & \% acumulado \\
\hline 1 & 5.044 & 45.854 & 45.854 \\
2 & 1.191 & 10.831 & 56.685 \\
3 & 0.774 & 7.032 & 63.717 \\
4 & 0.733 & 6.661 & 70.379 \\
5 & 0.627 & 5.696 & 76.074 \\
6 & 0.595 & 5.407 & 81.481 \\
7 & 0.518 & 4.713 & 86.194 \\
8 & 0.437 & 3.970 & 90.164 \\
9 & 0.409 & 3.715 & 93.879 \\
10 & 0.380 & 3.451 & 97.330 \\
11 & 0.294 & 2.670 & 100 \\
\hline Fuente: Elaboración propia & \multicolumn{3}{|c}{}
\end{tabular}

De acuerdo con los datos, los primeros tres autovalores tienen los siguientes valores: $\lambda_{1}=5.044 \lambda_{2}=$ 1.191 y $\lambda_{3}=0.774$. Por tanto:

$$
\frac{\lambda_{1}-\lambda_{2}}{\lambda_{2}-\lambda_{3}}=9.24>5
$$

Lo que permite tener por cumplido este criterio de evidencia del supuesto de unidimensionalidad. También se observa que el primer autovalor explica el $45.854 \%$ de la varianza total, por lo que se cumple el criterio de Carmines y Zeller (1979, como se cita en Burga, 2006).

De esta manera, se concluye que el instrumento de medida utilizado en la investigación evidencia unidimensionalidad.

\subsection{Contraste de las hipótesis}

La tabla X muestra los estadísticos descriptivos de la variable "ansiedad matemática" para la muestra total.

Tabla X. Estadísticos descriptivos de la variable "ansiedad matemática"

\begin{tabular}{lccccc}
\hline & N & Mínimo & Máximo & Media & Desv. típ. \\
\hline Ansiedad matemática & 3725 & 11.00 & 55.00 & 30.9487 & 9.44558 \\
N válido (según lista) & 3725 & & & & \\
\hline
\end{tabular}

Las diferencias en ansiedad matemática entre hombres ( $M=28.75$, $D S=9.28$ ) y mujeres $(M=32.97, D S=9.12)$ fue significativa $(\mathrm{T}(3725)=13.971, \mathrm{p}<0.05, \mathrm{~d}=0.458)$. De acuerdo con estos resultados las mujeres son más ansiosas que los hombres y el efecto de la variable sexo es de tamaño mediano.

El ANOVA señaló diferencias significativas entre los niveles educativos $(F(4.3720)=22.92, p<0.001)$. Las pruebas a posteriori (Bonferroni, alfa=0.05) indicaron que los niveles 7 a 9 no difirieron entre sí, pero fueron significativamente distintos de los niveles 10 y 11, que a su vez no difirieron entre ellos. 


\section{Discusión y Conclusión}

La investigación se enfocó en el tema de la ansiedad matemática en la educación secundaria oficial costarricense, con el objetivo general de medir el nivel de ansiedad matemática de los estudiantes y establecer si existen diferencias en la magnitud de esa variable por género o según el nivel educativo.

Los resultados indican que un porcentaje muy alto de los estudiantes, cercano al 78\%, muestra un nivel de ansiedad matemática medio, bajo o muy bajo. Este hallazgo de la investigación es, en cierto sentido, sorprendente en un país en el cual el rendimiento académico en matemática en la educación media es bajo y en el que se asume que la aceptación social de la matemática es baja.

La investigación devela también que las mujeres tienen niveles de ansiedad matemática más altos que los hombres, resultado que coincide con los obtenidos en otras investigaciones (Hembree, 1990; Ma, 1999; Gardner, 1997; Gil, Blanco y Guerrero, 2006) y los reportados por Pérez-Tyteca et al. (2013) con base en Fennema y Sherman (1976), Lafferty (1994), Vest (1998), Wigfield y Meece (1988) y Worley (1997).

No obstante estos resultados, aún no se han establecido explicaciones para tales diferencias. Autores como Perina (2002), Reyes (1984) y Martin (1994), citados por Pérez-Tyteca et al. (2013), sugieren que las diferencias podrían no ser reales sino que se trata de una asunto asociado a la medición de la ansiedad matemática, derivada del hecho de que las mujeres responden a los instrumentos de medida con mayor sinceridad sobre sus experiencias de ansiedad matemática que los hombres.

En consecuencia, las diferencias encontradas en el nivel de ansiedad matemática por género deben ser consideradas de manera conservadora, máxime si la magnitud del efecto resultó moderada.

Otro de los resultados obtenidos muestra la existencia de diferencias significativas en la variable ansiedad matemática según el nivel educativo a que pertenecen los estudiantes, distinguiendo niveles de ansiedad matemática distintos para los estudiantes del tercer ciclo y los estudiantes de la educación diversificada, resultando más ansiosos los últimos.

Tal como han señalado Pérez-Tyteca et al. (2013), con base en Seaman (1999), un efecto indirecto de la ansiedad matemática es que los estudiantes evitan llevar cursos con contenido matemático, lo que condiciona la elección de la carrera universitaria.

El hallazgo de la investigación resulta relevante porque muestra que, precisamente, los estudiantes más próximos a la elección de carrera universitaria son los que muestran los niveles de ansiedad matemática más alta. Aunque la magnitud del efecto también resultó moderada en este caso, el resultado obtenido debe servir para alertar a los docentes de matemática de la educación media y a otros actores educativos, como los orientadores o los directores de los colegios, de la conveniencia de procurar el desarrollo de programas de intervención educativa que atenúen los niveles de ansiedad matemática de los estudiantes de la educación diversificada.

Los resultados sugieren, al menos, dos líneas de investigación: profundizar en las posibles causales de las diferencias detectadas en el nivel de ansiedad matemática por género y de las que puedan explicar por qué se incrementa el nivel de ansiedad matemática cuando los estudiantes están en la educación diversificada.

\section{Referencias}

Burga, A. (2006). La unidimensionalidad de un instrumento de medición: perspectiva factorial. Revista de Psicología, 24(1), 53-80. Recuperado de http://revistas.pucp.edu.pe/index.php/psicologia/article/view/642

Cea, M. A. (1999). Metodología cuantitativa: estrategias y técnicas de investigación social. Madrid: Síntesis. 
Fennema, E. y Sherman, J. A. (1976). Fennema-Sherman mathematics attitude scales. Instruments designed to measure attitudes toward the learning of mathematics by males and females. JSAS Catalog of Selected Documents of Psychology, 6(31).

Gardner M. C. (1997). Changing math anxiety and attitudes with the use of graphics calculators: Differences by gender and age of student. Recuperado de http://www.eric.ed.gov/PDFS/ED412370.pdf

Gil, N., Blanco, L. y Guerrero, B. (2006). El papel de la afectividad en la resolución de problemas matemáticos. Revista de educación, 340, 551-569.

Gómez-Chacón, I. (2000). Matemática emocional: los afectos en el aprendizaje matemático. Madrid: Narcea.

Gómez-Chacón, I. (2010). Actitudes de los estudiantes en el aprendizaje de la matemática con tecnología. Enseñanza de las Ciencias, 28(2), 227-244.

Hembree, R. (1990). The nature, effects, and relief of mathematics anxiety. Journal for Research in Mathematics Education, 21(1), 33-46.

Hendel, D. (1980). Experiential and affective correlates of math anxiety in adult women. Psycology of Women Quartely, 5(2), 219-230.

Jiménez, K. y Montero, E. (2013). Aplicación del modelo de Rasch, en el análisis psicométrico de una prueba de diagnóstico en matemática. Revista digital Matemática, Educación e Internet, 13(1), 1-23.

Lozano, L. y De la Fuente-Solana, E. (2009). Diseño y validación de cuestionarios. En Pantoja-Vallejo (Coord.), Manual básico para la realización de tesinas, tesis y trabajos de investigación (pp. 251-274). España: EOS.

Ma, X. (1999). A meta-analysis of the relationship between anxiety toward mathematics and achievement in mathematics. Journal for Research in Mathematics Education, 30(5), 520-540.

Morales, P. (2008). Estadistica aplicada a las Ciencias Sociales. Madrid: Universidad Pontificia Comillas.

Morris, J. (1981). Mathematics anxiety: Teaching to avoid it. Mathematics Teacher, 74, 423-417.

Muñoz, J. y Mato, M. (2007). Elaboración y estructura factorial de un cuestionario para medir la "ansiedad hacia las Matemáticas" en alumnos de Educación Secundaria Obligatoria. Revista galego-portuguesa de Psicoloxía e Educación: revista de estudios e investigación en psicología y educación, 14, 221-231. Recuperado de: http://ruc.udc.es/dspace/handle/2183/7064

OCDE (2004). Informe PISA 2003: aprender para el mundo del mañana. España: Santillana.

Pérez-Tyteca, P. y Castro, E. (2011). La ansiedad matemática y su red de influencias en la elección de carrera Universitaria. Recuperado de

http://Funes.uniandes.edu.co/1831/1/402 Perez2011Laansiedad SEIEM13.pdf

Pérez-Tyteca, P. (2012). La ansiedad matemática como centro de un modelo causal predictivo de la elección de carreras. (Tesis doctoral). Universidad de Granada, España.

Pérez-Tyteca, P., Monje, J., y Castro, E. (2013). Afecto y matemáticas. Diseño de una entrevista para acceder a los sentimientos de alumnos adolescentes. Avances de Investigación en Educación Matemática, 4, 65-82.

Ripoll, J. (2011). La d de Cohen como tamaño del efecto. Recuperado de

http://clbe.wordpress.com/2011/10/26/la-d-de-cohen-como-tamano-del-efecto 
Rivas, T., Fierro, A., Jiménez, J. y Berrocal, C. (1998). Estudio de la estructura unidimensional de las escalas de bienestar personal y adaptación social. En Memorias del V Congreso de Evaluación Psicológica. Málaga. Recuperado de: http://www.eudemon.net/Estructura\%20de\%20las\%20Escalas\%20BP\%20y\%20AS.pdf

Tejedor, B., Santos, M. A., García-Orza, J., Carratalà, P. y Navas, M. (2009). Variables explicativas de la ansiedad frente a las matemáticas: un estudio de una muestra de 6o. de primaria. Anuario de psicología, 40(3), 345-355.

Valero, L. (1999) Evaluación de ansiedad ante exámenes: Datos de aplicación y fiabilidad de un cuestionario CAEX. Anales de Psicología, 15(2), 223-231. 